\title{
NAVIGATION IN ORAL AND MAXILLOFACIAL SURGERY: A BRIEF REVIEW OF LITERATURE
}

\author{
DR. DYNA ALBERT ${ }^{1}$, DR. JONES JAYABALAN ${ }^{2}$ \& PROF. DR. MR. MUTHUSEKHAR ${ }^{3}$ \\ ${ }^{1,2}$ Post Graduate Student, Department of Oral and Maxillofacial Surgery, Saveetha Dental College and Hospital, \\ Chennai, Tamil Nadu, India \\ ${ }^{3}$ Director of Programme, Department of Oral and Maxillofacial Surgery, Saveetha Dental College and Hospital,
}

Chennai, Tamil Nadu, India

\begin{abstract}
Cranio-maxillofacial skeleton is a complex entity due to its intricacy to the vital structures of skull base and cervical column. Throughout the years, surgeons have attempted to gain a better understanding of the region with the aid of the continuously evolving and innovating technologies. One such milestone in this horizon was the advent of Navigation Assisted Surgery. Having its roots in neurosurgery, it eventually gained acceptance and popularity in surgeries related to the cranio-maxillofacial region. The principle behind Navigation Assisted Surgery is similar to that of a Global Positioning System (GPS) with three primary components: the localizer, instrument or surgical probe and patient radiographic image. The two types of navigation system currently popular are the optical and electromagnetic systems. While optical system uses cameras fitted with Infrared (IR) filters fitted in front of the camera lens, electromagnetic system utilizes the electromagnetic field and both these systems are not without their pros and cons. In the craniomaxillofacial region, Navigation Assisted Surgery is now being utilized for a range of procedures beginning routine dentoalveolar surgeries to complex skull base surgeries. In this review, we aim to briefly discuss the history, principle, types and clinical applications of Navigation Assisted Surgery in oro-maxillofacial region.

KEYWORDS: Craniofacial surgery, Oral and maxillofacial surgery, Navigation assisted surgery\&Navigation surgery
\end{abstract}

Received: Jun 06, 2020; Accepted: Jun 26, 2020; Published: Jul 16, 2020; Paper Id.: IJMPERDJUN2020346

\section{INTRODUCTION}

The complexity of maxillofacial skeleton and its intricate relation to the vital structures of the skull base and vertebral column has made the field of maxillofacial surgery a unique surgical specialty(1). Irrespective of the type of deformity (developmental, congenital or acquired) in the maxillofacial skeleton, restoration of both form and function is of ineludible importance. The re-establishment of facial symmetry and esthetics becomes as prudent in treating these deformities(2). In the past, the 2 dimensional representation of the 3 dimensional face had made surgical precision and outcome extremely cumbersome, technique sensitive and sometimes irreproducible even in the hands of an experienced surgeon(3). With the rapid evolution of imaging and diagnostic technology, the achievement of these goals has not only become realistic but also less time consuming with reproducible successful results in trained hands(4).

The concept of Navigation Surgery was first introduced in Neurosurgical practice by Watanabe in 1987. The advent of Computed Tomography (CT) Imaging was the foundation upon which Watanabe et al proposed their new system of surgery which introduced the idea of CT stereotaxis into conventional open intracranial microsurgery. They stated that the technology enabled the surgeon to accurately identify and delineate the surgical 
site by obtaining real-time positional information in situ translated directly back onto CT images(5).

From the time of its advent, it took nearly 10 years for Navigation Assisted Surgery to set its foot into Craniomaxillo-facial surgery. It was not until early 2000s that surgeons began experimenting Navigation Technology in the maxillofacial region. In 2002, Schmelzeisen et al and Gellrich NC extended the use of this technology in the resection of ankylotic mass in TMJ ankylosis and orbital reconstruction surgeries respectively. They reported the safety, specificity, reliability and accuracy of this system in surgically managing the deformities of maxillofacial region. This minimally invasive system also allows lesser operating time with least surgical morbidities and complications. The accuracy of navigation assisted surgery is less than $1-2 \mathrm{~mm} .(6,7)$.

\section{SURGICAL NAVIGATION AND GPS SYSTEM}

A better understanding of the surgical navigation system can be obtained by paralleling it with the Global Positioning System (GPS) that is used by automobiles. It has three primary components: the localizer, instrument or surgical probe and patient radiographic image. The localizer of navigation system is analogous to the space satellite of GPS system. The instrument or surgical probe is analogous to the track waves emitted by the GPS unit in the automobile and the patient CT scan images which is analogous to the road map of GPS(8). The main advantage of computer assisted navigation surgery is the pre-operative planning of the surgical procedure with the help of CT images obtained from the patient. This comes in handy in complex cranio-facial surgeries which help increase intra-operative accuracy and decrease operating time. To do this, spatial linking of the patient, surgical instrument to the patient images is of prime importance(9).

\section{TYPES OF NAVIGATION SYSTEM}

The two types of navigation system currently available are the optical and electromagnetic systems. Though, both these systems accomplish the same task, the technology used in assisting the surgeon is not the same in these systems(10-12).

- Optical or Infrared Tracking System: To put it in simple terms, it monitors a defined measurement of space using cameras which are equipped with infrared (IR) filters fitted in front of camera lens and IR LEDs around the lens to illuminate the measurement space with IR light. These LEDs are fixed to the head of the patient and to the hand held probe.The wireless nature of this system is a great boon. The biggest disadvantage of this system is that it requires a clear line of vision between the patient, the instrument trackers and the optical cameras(10-12). This restricts the use of this system in certain navigational surgeries. Though the system is known for its linearity, accuracy and stability, there is the possibility of inaccuracies due to camera lens, image distortion and improper handling $(3,9,12,13)$.

- Electromagnetic Tracking System:As the name suggests, these systems use electromagnetic field. Similarly, the reference points carried on a device are attached to the head of the patient and a wired instrument that will be used by the surgeon in the surgical area of interest. The primary disadvantage of this system is the interference caused when metallic objects are in operatory field $(12,14,15)$. This has made the system less desirable in most surgical procedures. This system also requires wired connection for the trackers and an electromagnetic field generator which are an added bane to this system(10,12,14,16). However, this system does not require clear line of sight between its components which is its biggest boon $(14,16,17)$. Also, the sensing coils in this system are in close proximity to the tip of the tracked instrument thereby also being closer to the surgical area of interest. This makes tracking inside the human body with a flexible instrument possible. Nowadays, the effect on the accuracy of other 
metals in the presence of the field generator has been lowered by the miniaturization of the sensor coils(12).

\section{REGISTRATION IN NAVIGATION SURGERY}

Registration of the patient's anatomical structures is a crucial step in navigation assisted surgery and dictates the accuracy and outcome of the procedure. They can be done by one of the following methods:

- Marker Based Registration: Uses markers which are recognizable on the patient's pre-operative images and easily identifiable on the patient in intra-operative field during the surgery. These markers can be skin adhesive markers, referencing dental splints and bone-implant screws(18-20).

- Marker Free Registration: This type of referencing does not rely on markers but directly depend on the patient's craniofacial anatomy. Registration can be done using skeletal or soft tissue references. Skeletal reference points should be stable bony landmarks which are apparent on the CT images. Soft tissue referencing can be done by randomly matching the facial skin surface to the corresponding soft tissue points on the CT or MRI images with the help of LASER(21-23).

- Hybrid Registration: This is a combination of the marker free and marker based registration systems $(24,25)$.

- To put it simply, registration overlies one set of image data over another set of data and utilizes CT and/ or MRI imaging for the purpose.

\section{CLINICAL APPLICATIONS OF NAVIGATION ASSISTED SURGERY IN OMFS}

\section{Maxillofacial Trauma}

Maxillofacial trauma is one of the most commonly encountered scenarios in maxillofacial practice. Reports from various populations suggest mid-facial trauma involving zygomatico-maxillary complex and orbit to be the most commonly sustained traumatic injuries of the face. Trauma to these area compromise both function and facial esthetics. The reestablishment of facial width, facial height and facial prominences is the pinnacle in the management of these fractures. Achieving these treatment goals becomes easy with navigation assisted surgery. Since most of the injuries to mid-face are unilateral, projection of the unaffected side over the traumatized area aids the surgeon in providing superior results with lesser operating time and surgical complications(26,27).

\section{Orthognathic Surgery}

The main advantage of using navigation in orthognathic surgery is the decreased rate of surgical morbidities. Being an elective esthetic procedure, surgical morbidities like nerve damage are undesirable complications. With the help of navigation, the surgeon can better plan and execute the surgical treatment with ease and reliability. The pre-surgical planning and model surgery can be mimicked intra-operatively with help of navigation to provide the superior esthetic and functional results(28-30).

\section{Dentoalveolar Surgery}

Even routine dentoalveolar surgeries can sometimes pose surgical challenges owing to its obscure anatomical position or its proximity to vital structures. Determining the location of deeply impacted tooth is an important step in the surgical planning as most of the surgeons time is consumed intra-operatively in this process and can deter the confidence of the surgeon and tire them leading to more post-operative complications. With the help of navigation it is possible to determine 
the spatial position of the tooth with accuracy and the gives its relation to the adjacent vital structures in real-time. Though not recommended for routine cases, in complicated and difficult impaction surgeries navigation can aid in decreasing surgical time, enhancing surgeon's confidence and reducing post-operative complication(31-34).

\section{Removal of Forgein Bodies}

Prior to their surgical removal, foreign bodies inside the maxillofacial skeleton need to be assessed with respect to its position and relation to vital structures. This is especially critical in case of blast injuries or gunshot wounds where the normal anatomy is disfigured. As is already known, navigation surgery can help predict the accurate position in 3D space and aid in their removal. But the soft tissue architecture during the procedure cannot be accounted for with navigation and is an unfortunate limitation(35-37).

\section{Skull Base Surgery}

Skull base surgeries are one among the most critical to perform and require extensive knowledge of the anatomy. Even in experienced hands, unexpected complications may arise. Surgeries of temporomandibular joint(TMJ) fall in this category and must be carefully assessed especially when associated with tumors in the region. In the past, tumors of middle cranial fossa and skull base were considered inoperable due to their limited access and risk of haemorhage. Navigation technology makes skull base surgeries quicker and more reliable by dynamic display of the operating site thereby reducing the risk and increasing the quality and reliability of the surgery $(7,38,39)$.

\section{CONCLUSIONS}

Navigational surgery continues to evolve and though there are limitations of its use in dynamic skeletal structures like the mandible at present, the future of navigation assisted surgery is still full of hope.

\section{REFERNCES}

1. Gruber RP, Levine SM, Levine JP. Facial topography: clinical anatomy of the face. Plast Reconstr Surg. 2013 Jul;132(1):249.

2. Manson PN, Hoopes JE, Su CT. Structural pillars of the facial skeleton: an approach to the management of Le Fort fractures. Plast Reconstr Surg. 1980 Jul;66(1):54-62.

3. Hassfeld S, Mühling J, Zöller J. Intraoperative navigation in oral and maxillofacial surgery. Int J Oral Maxillofac Surg. 1995 Feb;24(1 Pt 2):111-9.

4. Diagnostic Imaging - PubMed [Internet]. [cited 2020 May 23]. Available from: https://pubmed.ncbi.nlm.nih.gov/22516558/?from_term=development+diagnostic+imaging\&from_pos=2

5. Watanabe E, Watanabe T, Manaka S, Mayanagi Y, Takakura K. Three-dimensional digitizer (neuronavigator): new equipment for computed tomography-guided stereotaxic surgery. Surg Neurol. 1987 Jun;27(6):543-7.

6. Gellrich N-C, Schramm A, Hammer B, Rojas S, Cufi D, Lagrèze W, et al. Computer-assisted secondary reconstruction of unilateral posttraumatic orbital deformity. Plast Reconstr Surg. 2002 Nov;110(6):1417-29.

7. Schmelzeisen R, Gellrich N-C, Schramm A, Schön R, Otten J-E. Navigation-guided resection of temporomandibular joint ankylosis promotes safety in skull base surgery. J Oral Maxillofac Surg Off J Am Assoc Oral Maxillofac Surg. 2002 Nov;60(11):1275-83.

8. Kumar V, Gour S, Chaube R, Chaube R, Gupta A, Chaube S. Surgical Navigation in Oral and Maxillofacial Surgery: A Review. Clin Res Open Access. 2018 Mar 9;4:1-6. 
9. Galloway RL Jr. Medical Imaging 2003: Visualization, Image-Guided Procedures, and Display. 2003 May 1 [cited 2020 May 24];5029. Available from: http://adsabs.harvard.edu/abs/2003SPIE.5029.....G

10. Intraoperative imaging guided delineation and localization of regions of surgical interest : feasibility study| Semantic Scholar [Internet]. [cited 2020 May 24]. Available from: https://www.semanticscholar.org/paper/Intraoperative-imaging-guideddelineation-and-of-of-Katisko/1c6d81a302e2395599a51a4955edecf3fbe3bcb0

11. Koivukangas T, Katisko JP, Koivukangas JP. Technical accuracy of optical and the electromagnetic tracking systems. SpringerPlus [Internet]. 2013 Mar 8 [cited 2020 May 24];2. Available from: https://www.ncbi.nlm.nih.gov/pmc/articles/PMC3622743/

12. Birkfellner W, Watzinger F, Wanschitz F, Ewers R, Bergmann H. Calibration of tracking systems in a surgical environment. IEEE Trans Med Imaging. 1998 Oct;17(5):737-42.

13. Vahala E, Ylihautala M, Tuominen J, Schiffbauer H, Katisko J, Yrjänä S, et al. Registration in interventional procedures with optical navigator. J Magn Reson Imaging JMRI. 2001 Jan;13(1):93-8.

14. Accuracy Assessment Protocols for Electromagnetic Tracking Systems - PubMed [Internet]. [cited 2020 May 24]. Available from: https://pubmed.ncbi.nlm.nih.gov/12894982/

15. Schicho K, Figl M, Donat M, Birkfellner W, Seemann R, Wagner A, et al. Stability of miniature electromagnetic tracking systems. Phys Med Biol. 2005 May 7;50(9):2089-98.

16. Chung AJ, Edwards PJ, Deligianni F, Yang G-Z. Freehand Cocalibration of Optical and Electromagnetic Trackers for Navigated Bronchoscopy. In: Yang G-Z, Jiang T-Z, editors. Medical Imaging and Augmented Reality. Berlin, Heidelberg: Springer; 2004. p. 320-8. (Lecture Notes in Computer Science).

17. Comparison of Magnetic Tracking and Optical Tracking by Simultaneous Use of Two Independent Frameless Stereotactic Systems - PubMed [Internet]. [cited 2020 May 24]. Available from: https://pubmed.ncbi.nlm.nih.gov/16234678/

18. Reconstruction of Orbital Wall Defects With Calcium Phosphate Cement: Clinical and Histological Findings in a Sheep Model - PubMed [Internet]. [cited 2020 May 24]. Available from: https://pubmed.ncbi.nlm.nih.gov/16965899/?from_term=reconstruction+of+orbital+wall+defect+with+calcium +phosphate+ cement\&from_pos $=1$

19. A Comparison of Computer-Aided Surgery Registration Methods for Endoscopic Sinus Surgery - PubMed [Internet]. [cited 2020 May 24]. Available from: https://pubmed.ncbi.nlm.nih.gov/16539295/

20. Point-to-point Registration With Mandibulo-Maxillary Splint in Open and Closed Jaw Position. Evaluation of Registration Accuracy for Computer-Aided Surgery of the Mandible - PubMed [Internet]. [cited 2020 May 24]. Available from: https://pubmed.ncbi.nlm.nih.gov/22079336/

21. Hoffmann J, Westendorff C, Leitner C, Bartz D, Reinert S. Validation of 3D-laser surface registration for image-guided cranio-maxillofacial surgery. J Cranio-Maxillo-fac Surg Off Publ Eur Assoc Cranio-Maxillo-fac Surg. 2005 Feb;33(1):13-8.

22. Marmulla R, Lüth T, Mühling J, Hassfeld S. Markerless laser registration in image-guided oral and maxillofacial surgery. $J$ Oral Maxillofac Surg Off J Am Assoc Oral Maxillofac Surg. 2004 Jul;62(7):845-51.

23. Laser Surface Registration for Lateral Skull Base Surgery - PubMed [Internet]. [cited 2020 May 24]. Available from: https://pubmed.ncbi.nlm.nih.gov/16015497/?from_term=laser+surface+registration\&from_pos=1

24. Hong J, Matsumoto N, Ouchida R, Komune S, Hashizume M. Medical navigation system for otologic surgery based on hybrid registration and virtual intraoperative computed tomography. IEEE Trans Biomed Eng. 2009 Feb;56(2):426-32. 
25. A Hybrid Method to Improve Target Registration Accuracy in Surgical Navigation - PubMed [Internet]. [cited 2020 May 24]. Available from: https://pubmed.ncbi.nlm.nih.gov/25761496/?from_term=hybrid+method+to+improve+target+registration\&from_pos=1

26. “Mirroring” Computational Planning, Navigation Guidance System, and Intraoperative Mobile C-arm Cone-Beam Computed Tomography With Flat-Panel Detector: A New Rationale in Primary and Secondary Treatment of Midfacial Fractures? PubMed [Internet]. [cited 2020 May 24]. Available from: https://pubmed.ncbi.nlm.nih.gov/21185639/

27. Manson PN, Clark N, Robertson B, Slezak S, Wheatly M, Vander Kolk C, et al. Subunit principles in midface fractures: the importance of sagittal buttresses, soft-tissue reductions, and sequencing treatment of segmental fractures. Plast Reconstr Surg. 1999 Apr;103(4):1287-306; quiz 1307.

28. Cross-sectional Tomography in Evaluation of Patients Undergoing Sagittal Split Osteotomy - PubMed [Internet]. [cited 2020 May 24]. Available from: https://pubmed.ncbi.nlm.nih.gov/1447604/?from_term=hallikainen+D\&from_pos=9

29. Kang S-H, Kim M-K, Choi Y-S, Park W, Lee S-H. Navigation-assisted intraoral vertical ramus osteotomy. J Oral Maxillofac Surg Off J Am Assoc Oral Maxillofac Surg. 2011 Mar;69(3):931-4.

30. Noleto JW, Marchiori E, Da Silveira HM. Evaluation of mandibular ramus morphology using computed tomography in patients with mandibular prognathism and retrognathia: relevance to the sagittal split ramus osteotomy. J Oral Maxillofac Surg Off J Am Assoc Oral Maxillofac Surg. 2010 Aug;68(8):1788-94.

31. Lyu K, Yang RT, Zhou HH, Li Z, Li ZB, Yang XW. [Clinical retrospective study of navigation guided impacted maxillary supernumerary tooth extraction]. Zhonghua Kou Qiang Yi Xue Za Zhi Zhonghua Kouqiang Yixue Zazhi Chin J Stomatol. 2018 Feb 9;53(2):103-6.

32. Navigation-Guided Extraction of Impacted Supernumerary Teeth: A Case Report - PubMed [Internet]. [cited 2020 May 24]. Available from: https://pubmed.ncbi.nlm.nih.gov/28279686/

33. Retrieval of a Displaced Third Molar Using Navigation and Active Image Guidance - PubMed [Internet]. [cited 2020 May 24]. Available from: https://pubmed.ncbi.nlm.nih.gov/20116728/

34. Iwai T, Matsui Y, Hirota M, Tohnai I. Endoscopic removal of a maxillary third molar displaced into the maxillary sinus via the socket. J Craniofac Surg. 2012 Jul;23(4):e295-296.

35. Lee TYT, Zaid WS. Broken dental needle retrieval using a surgical navigation system: a case report and literature review. Oral Surg Oral Med Oral Pathol Oral Radiol. 2015 Feb;119(2):e55-59.

36. Nezafati S, Shahi S. Removal of broken dental needle using mobile digital C-arm. J Oral Sci. 2008 Sep;50(3):351-3.

37. Removal of a Foreign Body From the Skull Base Using a Customized Computer-Designed Guide Bar - PubMed [Internet]. [cited 2020 May 24]. Available from: https://pubmed.ncbi.nlm.nih.gov/19683935/

38. He D, Yang C, Shen G, Chen M, Yang X, Huang D, et al. Navigation-guided resection for a tenosynovial giant cell tumor involving the temporomandibular joint and skull base. J Craniofac Surg. 2012 Mar;23(2):521-3.

39. Navigation-guided Resection With Immediate Functional Reconstruction for High-Grade Malignant Parotid Tumour at Skull Base - PubMed [Internet]. [cited 2020 May 24]. Available from: https://pubmed.ncbi.nlm.nih.gov/19473816/ 